\title{
Heart and vascular remodeling in essential hypertension and type 2 diabetes is dependent on genetic polymorphisms
}

\author{
Anna Shalimova \\ Kharkiv National Medical University, Kharkiv 61022, Ukraine. \\ Correspondence to: Dr. Anna Shalimova, Kharkiv National Medical University, 4 Nauky Avenue, Kharkiv 61022, Ukraine. \\ E-mail: annashalimova@yandex.ua
}

How to cite this article: Shalimova A. Heart and vascular remodeling in essential hypertension and type 2 diabetes is dependent on genetic polymorphisms. Vessel Plus 2017;1:84-90.

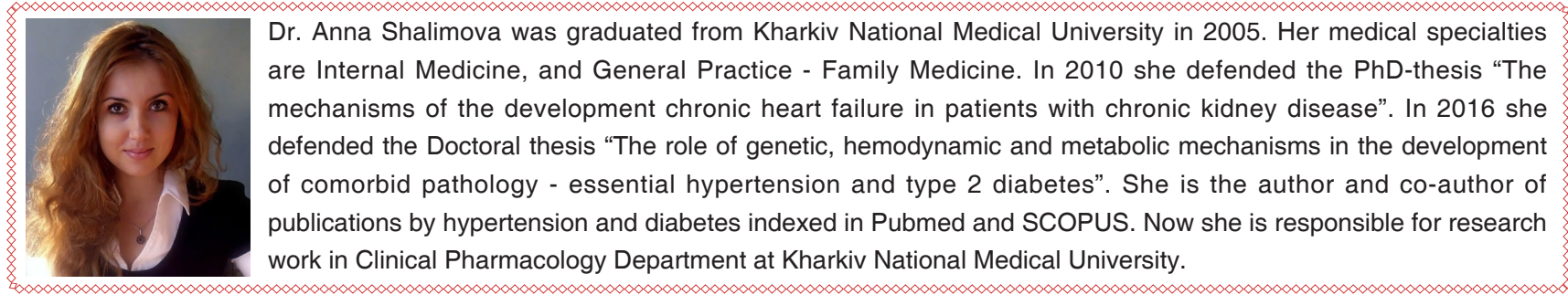

Article history:

Received: $22-10-2016$

Accepted: 29-12-2016

Published: 27-06-2017

\section{Key words:}

Essential hypertension, type 2 diabetes comorbidity, genetic polymorphism, heart and vascular remodeling

\begin{abstract}
Aim: To study heart and vascular remodeling in essential hypertension (EH) and concomitant type 2 diabetes mellitus (DM2) with respect to genetic polymorphism of the angiotensin II receptor type 1 (AGTR1) gene and peroxisome proliferator-activated receptor- $\gamma 2$ (PPAR 2$)$. Methods: Biochemical blood analysis, echocardiographic evaluation of mitral diastolic blood flow and tissue Doppler spectral modes, reactive hyperemia, color Doppler mapping. Results: Patients with $A / C$ and $C / C$ genotypes of the $A G T R 1$ gene had higher blood pressure, more pronounced metabolic disorders, a larger left ventricle (LV), higher myocardial mass index left ventricle, and a greater intima media thickness (IMT), with a lower rate of endotheliumdependent vasodilation (EDVD) compared to the $A / A$ genotype. Patients with the Pro/Pro genotype of $P P A R \gamma 2$ had higher levels of blood pressure, larger LVs, greater IMT, pulse wave velocity, and a lower rate of EDVD compared to the Pro/Ala and Ala/Ala genotypes. Patients with the Pro/Pro genotype had significantly more pronounced dyslipidemia and insulin resistance than patients with other $P P A R \gamma 2$ genotypes. Conclusion: The polymorphism of genetic markers $A G T R 1$ and PPAR 2 in patients with EH and concomitant DM2 was associated with the development of comorbidity. Different genotypes of specific genes alter the severity of cardiovascular remodeling and metabolic disorders.
\end{abstract}

This is an open access article licensed under the terms of Creative Commons Attribution 4.0 International License (https://creativecommons.org/licenses/by/4.0/), which permits unrestricted use, distribution, and reproduction in any medium, as long as the original author is credited and the new creations are licensed under the identical terms.

For reprints contact: service@oaepublish.com

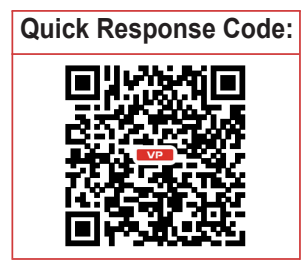




\section{INTRODUCTION}

There are a large number of patients with essential hypertension $(\mathrm{EH})$ who also have type 2 diabetes mellitus (DM2), thus meriting further research into this problem. It is known that $\mathrm{EH}$ and DM2 have many common pathogenetic mechanisms that affect the development of comorbidity. According to many researchers, the most important predictors of $\mathrm{EH}$ and DM2 are hereditary risk factors. ${ }^{[1-3]}$ However, there are controversial views on the role of gene expression and genetic polymorphisms in the development and course of diseases in different populations of patients, as well as their influence on the effectiveness of drug therapy.

Some studies have shown that the predisposing cause of $\mathrm{EH}$ can be mutational alleles of the angiotensin II receptor (AGTR1) gene; angiotensin is a powerful vasoconstrictor, thus playing a role in the pathogenesis of EH. ${ }^{[4,5]}$ Angiotensin II (AT-II) receptor type 1, which is located on the vascular endothelium, mediates the main cardiovascular effects of angiotensin, including the induction of insulin-like growth factor and endothelin-1. The induction of cell growth is also mediated through AGTR1 ${ }^{[6]}$ A number of investigations have reported that AGTR1 polymorphisms may lead to changes in the regulation of vascular tone and proliferation of vascular wall elements. ${ }^{[7]}$

Hyperinsulinemia and insulin resistance (IR) are the factors that determine the frequency of cardiovascular complications in DM2. ${ }^{[8-10]}$ Despite the fact that IR has a clearly identified genetic predisposition, its underlying genetic disorders have still not been identified.

There has been extensive research into the polymorphisms of peroxisome proliferator-activated receptors (PPAR), which are transcription factors that control the activity of many genes, as well as regulating lipid and carbohydrate metabolism. ${ }^{[11,12]}$ Here we focused on PPAR 2, which is almost exclusively located in adipose tissue, and plays a vital role in controlling adipogenesis and circulation of fatty acids.

It has been established that genetic polymorphisms of $P P A R \gamma 2$ are different in diverse populations, and data on the effect of PPAR 2 on the development of IR are quite controversial. Therefore, there is a continued interest by scientists into the study of PPAR 2 polymorphisms in the development of IR and other pathological processes, and the presence of conflicting data regarding its role in different populations justifies the ongoing research into the Ukrainian population of patients with comorbid disorders.

Our aim was to study heart and vascular remodeling in patients with $\mathrm{EH}$ and concomitant DM2 according to the genetic polymorphisms of AGTR1 and PPAR 2 .

\section{METHODS}

The authors examined 320 patients with $\mathrm{EH}$ stage II grade 2 and moderate, sub-compensated DM2 (the main group); 90 patients with $\mathrm{EH}$ stage II grade 2 without DM2 (the comparison group); and 31 healthy individuals (the control group).

In this study, using standard biochemical methods on the patients, we defined venous blood glucose concentration, glycosylated hemoglobin (HbA1c), and insulin levels. IR was determined using the homeostasis model assessment index (HOMA-IR). Ultrasound examinations were performed on a cardiac ultrasound scanner ("ULTIMA RA» firm "RADMIR", Ukraine) in one-, two-dimensional and Doppler modes with color mapping by conventional methods. The following measurements were made: volumes of the left atrium (LA) and right atrium (RA); end-systolic diameter (ESD) and end-diastolic diameter (EDD) of the left ventricle (LV); end-diastolic pressure (EDP) in the LV; left ventricular ejection fraction (EF); index of relative wall thickness (IRWT); and the myocardial mass index (MMI) of the LV. Diastolic function of the LV was assessed by studying the blood flow in the pulmonary artery and transmitral diastolic flow in the pulsed Doppler mode with the following parameters: maximum rate of early $L V$ filling $(E)$; maximum speed of late (atrial) LV filling (A); the ratio of the maximum velocity of early and late LV filling (E/A); LV isovolumic relaxation time (IVRT); deceleration time (DT) of early diastolic flow velocity; average pulmonary artery pressure (PAP), according to Kitabatake; the ratio of peak $E$ and e on the mitral valve in the spectral and tissue Doppler $(E / e)$. For studying endothelial function, the degree of endothelium-dependent vasodilation (EDVD) in reactive hyperemia was determined in all patients. Investigations were carried out using a broadband linear transducer 5-12 MHz Doppler color mapping with three readings being taken arteries at 15 -min intervals between samples on the left and right brachial arteries, according to the method of Celermajer DS (a modification of the method by Ivanova OV). Normally, the maximum vasodilation of the brachial artery should exceed $10 \%$ of the original diameter. Simultaneously, we measured the intima media thickness (IMT) of the carotid artery (CA $2 \mathrm{~cm}$ proximal to the bifurcation of the common carotid artery). Pulse wave velocity (PWV) of the CA was determined using the W-Trackmethod (method of phase tracking, patented scanner developers). Determining the PWV of the abdominal aortic (AA) (on the left subclavian artery to the femoral 
artery) was performed using a phased transducer with a frequency of 2-4 MHz. An A1166C polymorphism of the AGTR1 gene and a Pro12Ala polymorphism of the PPAR 2 gene were assessed by the molecular genetic method. Three genotypes of the AGTR1 gene $(A / A, A / C$ and $C / C)$ were identified, along with three genotypes of the PPAR 2 gene (Pro/Pro, Pro/Ala and Ala/Ala). Processing of statistical data was performed using the software package "Statistics for Windows 6.0 ". The values are presented as the average value of parameters $(\mathrm{M})$ and standard error $(\mathrm{m})$.

The study protocol was approved by the Ethics Committee of the Kharkiv National Medical University. All participants were informed about the aim of the study and signed a written consent form.

\section{RESULTS}

Evaluation of the $A 1166 C$ polymorphism of the AGTR1 gene in patients with comorbidity of $\mathrm{EH}$ and $\mathrm{DM} 2$, compared to the distribution of alleles and genotypes in healthy individuals, and in patients with $\mathrm{EH}$ but without DM2, showed that $61.6 \%$ of patients with $\mathrm{EH}$ and DM2, and $57.8 \%$ of patients with $\mathrm{EH}$ without DM2, had $A / C$ and $C / C$ genotypes of $A G T R 1$, which have been shown to be associated with cardiovascular complications by some researchers. ${ }^{[9,10]}$ The genotypes of the main group $(P<0.01)$ and the comparison group $(P<0.05)$ were significantly different from the control group. The $C$ allele was established in $33.1 \%$ of patients with $\mathrm{EH}$ and DM2, and $31.1 \%$ of patients with $\mathrm{EH}$ without DM2; in the control group, the $C$ allele was significantly less present $(P<0.05)$ [Table 1].

In the next step of the study, hemodynamic and metabolic parameters in patients with comorbidity of $\mathrm{EH}$ and DM2, in different polymorphism variants of the AGTR1 gene, were compared [Table 2].

Patients with $A / C$ and $C / C$ genotypes of the AGTR1 gene had higher blood pressure $(P<0.001)$ compared to the $A / A$ genotype. These patients also had significantly larger LV and myocardial mass index left ventricle (MMILV), and a greater IMT, with a lower rate of EDVD. Patients with the indicated genotypes also had significantly more pronounced metabolic disorders than patients with the $A / A$ genotype. No significant differences in hemodynamic and metabolic parameters between the $C / C$ and $A / C$ genotypes were found [Table 2].

Given that $A / C$ and $C / C$ genotypes presented significantly different characteristics from the A/A genotype, with more severe disorders of echocardiographic and biochemical parameters, but were not significantly different from each other, in the next step of the study, patients with $A / C$ and $C / C$ genotypes were merged into a single group, namely the $A / C+C / C$ genotype.

For establishing associations of AGTR1 polymorphisms with cardiovascular remodeling, a comparative evaluation of echocardiographic parameters and indicators of the structural and functional state of the heart and blood vessels of the main group of patients, with different genotypes, was performed [Table 3]. It was found that patients with the $A / C+C / C$ genotype had significantly larger LV compared to the $A / A$ genotype $(P<0.01)$. Thus, patients with the $A / C+$ $C / C$ genotype also had significantly $(P<0.05)$ greater MMILV compared to the $A / A$ genotype. In addition, the $A / C+C / C$ genotype had significantly $(P<0.05)$ lower values of the diastolic function indicator $E / A$.

Assessment of the great vessels showed that IMT in the main group of patients with the genotype $A / C$ $+C / C$ was significantly $(P<0.001)$ greater than the genotype $A / A$ [Table 3]. The study did not reveal any significant differences in PWV values in the carotid artery or the abdominal aorta in the diverse AGTR1 genotypes. It should be noted that in patients with $\mathrm{EH}$ with concomitant DM2 and genotype $A / C+C / C$, the level of EDVD was significantly $(P<0.001)$ lower than the genotype $A / A$.

In the next step of the study, the Pro12Ala polymorphism of PPAR 2 was estimated and compared with the distribution of alleles and genotypes in healthy individuals and in patients with EH without DM2 [Table 4].

It was found that, in all study groups, patients with the Pro allele were predominant $(86.6 \%$ in the main group, $85.6 \%$ in the comparison group and $87.1 \%$ in

Table 1: The distribution of AGTR1 alleles and genotypes in the patients, $n(\%)$

\begin{tabular}{lccc}
\hline Indices & Main group $(\boldsymbol{n}=\mathbf{3 2 0})$ & Comparison group $(\boldsymbol{n}=\mathbf{9 0})$ & Control group $(\boldsymbol{n}=\mathbf{3 1})$ \\
\hline A allele & $214(66.9)^{*}$ & $62(68.9)^{*}$ & $25(80.6)$ \\
C allele & $106(33.1)^{*}$ & $28(31.1)^{*}$ & $6(19.4)$ \\
A/A genotype & $123(38.4)^{*}$ & $38(42.2)^{*}$ & $20(64.5)$ \\
$A / C$ genotype & $182(56.9)^{*}$ & $49(54.5)^{*}$ & $10(32.3)$ \\
C/C genotype & $15(4.7)$ & $3(3.3)$ & $1(3.2)$ \\
\hline
\end{tabular}

${ }^{*} P<0.05$ vs. the control group 
Table 2: Comparative evaluation of hemodynamic and metabolic parameters in patients of the main group depending on genotypes of the AGTR1 gene

\begin{tabular}{lccc}
\hline Indices & \multicolumn{3}{c}{ Main group $(\boldsymbol{n}=\mathbf{3 2 0})$} \\
\cline { 2 - 4 } & $\boldsymbol{A} / \boldsymbol{A}(\boldsymbol{n}=\mathbf{1 2 3})$ & $\boldsymbol{A} / \boldsymbol{C}(\boldsymbol{n}=\mathbf{1 8 2})$ & $\boldsymbol{C} \boldsymbol{C}(\boldsymbol{n}=\mathbf{1 5})$ \\
\hline SBP, $\mathrm{mmHg}$ & $166.041 \pm 0.261$ & $173.821 \pm 0.221^{*}$ & $172.401 \pm 0.621^{*}$ \\
DBP, mmHg & $99.461 \pm 0.181$ & $102.041 \pm 0.231^{*}$ & $5.331 \pm 0.851^{*}$ \\
EDD LV, cm & $4.911 \pm 0.031$ & $5.031 \pm 0.031^{*}$ & $3.421 \pm 0.095^{*}$ \\
ESD LV, cm & $3.221 \pm 0.031$ & $3.321 \pm 0.031^{*}$ & $146.611 \pm 3.791^{*}$ \\
MMI LV, g/m & $134.571 \pm 2.391$ & $143.931 \pm 3.09^{*}$ & $0.981 \pm 0.021$ \\
E/A & $0.950 \pm 0.021$ & $0.897 \pm 0.015^{*}$ & $6.661 \pm 0.291$ \\
E/e & $6.223 \pm 0.104$ & $6.411 \pm 0.251$ & $0.969 \pm 0.024^{*}$ \\
IMT, mm & $0.914 \pm 0.009$ & $0.953 \pm 0.007^{*}$ & $9.033 \pm 0.333$ \\
PWV of the CA, m/c & $8.736 \pm 0.097$ & $8.870 \pm 0.087$ & $9.109 \pm 0.354$ \\
PWV of the AA, m/c & $8.866 \pm 0.118$ & $9.013 \pm 0.099$ & $6.187 \pm 0.242^{*}$ \\
EDVD, \% & $6.657 \pm 0.077$ & $6.211 \pm 0.065^{*}$ & $7.201 \pm 0.025^{*}$ \\
blood glucose, mmol/L & $6.933 \pm 0.052$ & $7.046 \pm 0.0291^{*}$ & $7.104 \pm 0.015^{*}$ \\
HbA1c, \% & $6.993 \pm 0.044$ & $7.011 \pm 0.0361^{*}$ & $26.840 \pm 0.994^{*}$ \\
insulin, mcU/mL & $23.249 \pm 0.416$ & $24.982 \pm 0.291^{*}$ & $8.276 \pm 0.324^{*}$ \\
HOMA-IR & $7.272 \pm 0.130$ & $7.991 \pm 0.097^{*}$ & \\
\hline
\end{tabular}

${ }^{*} P<0.05$ Vs. the A/A genotypes. SBP: systolic blood pressure; DBP: diastolic blood pressure; EDD LV: end-diastolic diameter of left ventricle; ESD LV: end-systolic diameter of left ventricle; MMI LV: myocardial mass index of left ventricle; E/A: ratio of the maximum velocity of early and late left ventricle filling; E/e: ratio of peak e and $E$ on the mitral valve in the spectral and tissue Doppler; IMT: intima media thickness; PVW CA: pulse wave velocity by the carotid artery; PVW AA: pulse wave velocity by the abdominal aortic; EDVD: endothelium dependent vasodilation; HOMA-IR: homeostasis model assessment index

Table 3: Structural and functional state of heart and vessels in patients of the main group depending on genotypes of the AGTR1 gene

\begin{tabular}{lcc}
\hline \multirow{2}{*}{ Indices } & \multicolumn{1}{c}{ Main group $(\boldsymbol{n}=\mathbf{3 2 0})$} & $\boldsymbol{A} / \boldsymbol{C}+\boldsymbol{C} / \boldsymbol{C}(\boldsymbol{n}=\mathbf{1 9 7})$ \\
\cline { 2 - 3 } & $\boldsymbol{A} / \boldsymbol{A}(\boldsymbol{n = 1 2 3})$ & $5.040 \pm 0.030^{*}$ \\
\hline EDD LV, cm & $4.911 \pm 0.031$ & $3.326 \pm 0.027^{*}$ \\
ESD LV, cm & $3.221 \pm 0.031$ & $144.138 \pm 2.965^{*}$ \\
MMI LV, g/m & $134.571 \pm 3.391$ & $0.903 \pm 0.014^{*}$ \\
E/A & $0.951 \pm 0.021$ & $6.411 \pm 0.151$ \\
E/e & $6.223 \pm 0.104$ & $0.952 \pm 0.007^{*}$ \\
IMT, mm & $0.914 \pm 0.009$ & $8.883 \pm 0.084$ \\
PWV of the CA, m/c & $8.736 \pm 0.097$ & $9.020 \pm 0.095$ \\
PWV of the AA, m/c & $8.866 \pm 0.118$ & $6.180 \pm 0.062^{*}$ \\
EDVD, \% & $6.657 \pm 0.077$ & \\
\hline
\end{tabular}

${ }^{*} P<0.05$ vs. the A/A genotypes. EDD LV: end-diastolic diameter of left ventricle; ESD LV: end-systolic diameter of left ventricle; MMI LV: myocardial mass index of left ventricle; E/A: ratio of the maximum velocity of early and late left ventricle filling; E/e: ratio of peak e and $E$ on the mitral valve in the spectral and tissue Doppler; IMT: intima media thickness; PVW CA: pulse wave velocity by the carotid artery; PVW AA: pulse wave velocity by the abdominal aortic; EDVD: endothelium dependent vasodilation

Table 4: Distribution of PPAR $\gamma 2$ alleles and genotypes in the patients, $n(\%)$

\begin{tabular}{lccc}
\hline Indices & Main group $(\boldsymbol{n}=\mathbf{3 2 0})$ & Comparison group $(\boldsymbol{n = 9 0 )}$ & Control group $(\boldsymbol{n}=\mathbf{3 1})$ \\
\hline Pro allele & $277(86.6)$ & $77(85.6)$ & $27(87.1)$ \\
Ala allele & $43(13.4)$ & $13(14.4)$ & $4(12.9)$ \\
Pro/Pro genotype & $242(75.6)$ & $67(74.4)$ & $24(77.4)$ \\
Pro/Ala genotype & $71(22.2)$ & $21(23.3)$ & $6(19.4)$ \\
Ala/Ala genotype & $7(2.2)$ & $2(2.3)$ & $1(3.2)$ \\
\hline
\end{tabular}

the control group). It was also demonstrated that, in the main group and the comparison group, there were no significant differences in the frequency of different variants of the PPAR 2 genotype. In both these patient groups, the Pro/Pro genotype was predominant, with a frequency of $75.6 \%$ and $74.4 \%$, respectively. The homozygous genotype Ala/Ala was only found in $2.2 \%$ of the main group of patients and $2.3 \%$ of the comparison group of patients $(P>0.05)$. In the control group of patients, the Pro/Pro genotype was also prevalent (77.4\% of cases); Pro/Ala and Ala/Ala genotypes were found in $19.4 \%$ and $3.2 \%$ of control patients, respectively. A similar distribution of PPAR genotypes, according to other researchers ${ }^{[13]}$ was inherent in the European population.

Comparison of hemodynamic and metabolic parameters of patients with $\mathrm{EH}$ and concomitant DM2, in different variants of PPAR 2 polymorphisms, showed that patients with the Pro/Pro genotype of PPAR 2 had significantly $(P<0.01)$ higher levels of blood pressure; larger LV sizes; greater IMT and PWV, with a lower EDVD degree, compared to the Pro/Ala and Ala/Ala genotypes [Table 5]. In addition, patients with the Pro/Pro genotype had significantly more pronounced dyslipidemia $(P<0.01)$ and IR $(P<0.001)$ 
Table 5: Comparative evaluation of hemodynamic and metabolic parameters in patients of the main group depending on genotypes of PPAR $\gamma 2$

\begin{tabular}{lccc}
\hline \multirow{2}{*}{ Indices } & \multicolumn{3}{c}{ Main group $(\boldsymbol{n}=\mathbf{3 2 0})$} \\
\cline { 2 - 4 } & Pro/Pro & Pro/Ala & Ala/Ala \\
SBP, mmHg & $172.372 \pm 0.259$ & $165.775 \pm 0.351^{*}$ & $165.714 \pm 1.017^{*}$ \\
DBP, mmHg & $101.599 \pm 0.196$ & $99.338 \pm 0.232^{*}$ & $100.001 \pm 1113$ \\
EDD LV, cm & $5.024 \pm 0.026$ & $4.878 \pm 0.038^{*}$ & $4.856 \pm 0.084$ \\
ESD LV, cm & $3.316 \pm 0.024$ & $3.198 \pm 0.034^{*}$ & $3.149 \pm 0.072$ \\
MMI LV, g/m & $142.794 \pm 2.551$ & $136.553 \pm 2.983$ & $139.933 \pm 7.664$ \\
E/A & $0.924 \pm 0.013$ & $0.915 \pm 0.027$ & $0.943 \pm 0.158$ \\
E/e & $6.348 \pm 0.097$ & $6.108 \pm 0.193$ & $6.422 \pm 0.866$ \\
IMT, mm & $0.951 \pm 0.006$ & $0.900 \pm 0.011^{*}$ & $0.849 \pm 0.035^{*}$ \\
PWV of the CA, m/c & $8.910 \pm 0.075$ & $8.592 \pm 0.122^{*}$ & $8.301 \pm 0.301$ \\
PWV of the AA, m/c & $9.020 \pm 0.086$ & $8.874 \pm 0.146$ & $7.779 \pm 0.461^{* 0}$ \\
EDVD, \% & $6.155 \pm 0.056$ & $7.017 \pm 0.079^{*}$ & $6.959 \pm 0.241^{*}$ \\
blood glucose, mmol/L & $7.190 \pm 0.022$ & $6.968 \pm 0.029^{*}$ & $6.643 \pm 0.057^{*}$ \\
HbA1c, \% & $7.103 \pm 0.013$ & $6.939 \pm 0.058^{*}$ & $6.929 \pm 0.042^{*}$ \\
insulin, mcU/mL & $25.182 \pm 0.255$ & $21.906 \pm 1.526$ & $22.814 \pm 1.735$ \\
HOMA-IR & $8.039 \pm 0.083$ & $6.767 \pm 0.156^{*}$ & $6.722 \pm 0.484^{*}$ \\
\hline
\end{tabular}

${ }^{*} P<0.05$ vs. the Pro/Pro genotypes; ${ }^{\circ} P<0.05$ vs. the Pro/Ala genotypes. SBP: systolic blood pressure; DBP: diastolic blood pressure; EDD LV: end-diastolic diameter of left ventricle; ESD LV: end-systolic diameter of left ventricle; MMI LV: myocardial mass index of left ventricle; E/A: ratio of the maximum velocity of early and late left ventricle filling; E/e: ratio of peak e and $E$ on the mitral valve in the spectral and tissue Doppler; IMT: intima media thickness; PVW CA: pulse wave velocity by the carotid artery; PVW AA: pulse wave velocity by the abdominal aortic; EDVD: endothelium dependent vasodilation; HOMA-IR: homeostasis model assessment index

Table 6: Structural and functional state of the heart and vessels in the main group of patients depending on PPAR 2 genotypes

\begin{tabular}{lcc}
\hline \multirow{2}{*}{ Indices } & \multicolumn{2}{c}{ Main group $(\boldsymbol{n}=\mathbf{3 2 0})$} \\
\cline { 2 - 3 } & Pro/Pro $(\boldsymbol{n}=\mathbf{2 4 2})$ & Pro/Ala + Ala/Ala $(\boldsymbol{n}=\mathbf{7 8})$ \\
\hline EDD LV, cm & $5.024 \pm 0.026$ & $4.876 \pm 0.035^{*}$ \\
ESD LV, cm & $3.316 \pm 0.024$ & $3.194 \pm 0.032^{*}$ \\
MMI LV, g/m & $142.794 \pm 2.551$ & $133.215 \pm 2.799^{*}$ \\
E/A & $0.924 \pm 0.013$ & $0.917 \pm 0.028$ \\
E/e & $6.348 \pm 0.097$ & $6.136 \pm 0.190$ \\
IMT, mm & $0.951 \pm 0.006$ & $0.895 \pm 0.011^{*}$ \\
PWV of the CA, m/c & $8.910 \pm 0.075$ & $8.566 \pm 0.114^{*}$ \\
PWV of the AA, m/c & $9.020 \pm 0.086$ & $8.776 \pm 0.143$ \\
EDVD, \% & $6.155 \pm 0.056$ & $7.012 \pm 0.075^{*}$
\end{tabular}

${ }^{*} P<0.05$ vs. the Pro/Pro genotypes. EDD LV: end-diastolic diameter of left ventricle; ESD LV: end-systolic diameter of left ventricle; MM LV: myocardial mass index of left ventricle; E/A: ratio of the maximum velocity of early and late left ventricle filling; E/e: ratio of peak e and E on the mitral valve in the spectral and tissue Doppler; IMT: intima media thickness; PVW CA: pulse wave velocity by the carotid artery; PVW AA: pulse wave velocity by the abdominal aortic; EDVD: endothelium dependent vasodilation

than patients with other PPAR 2 genotypes.

However, the only significant difference in indicator levels, between the Ala/Ala and Pro/Ala genotypes was found in the PWV of the AA $(P<0.05)$. Given the fact that the Pro/Ala and Ala/Ala genotypes were significantly different from the Pro/Pro genotype, with the former genotypes collectively presenting less severe disorders of hemodynamic and metabolic indicators, but only differing from each other with respect to the PWV of the AA, and given the small percentage of patients with the homozygous Ala/Ala genotype, in the subsequent step, patients with the Ala/ Ala and Pro/Ala genotypes were merged into a single group, namely the Pro12Ala/Ala12Ala genotype.

Analysis of the differences of indicators in the structural and functional state of the heart showed that patients with the Pro12Ala/Ala12Ala genotype had significantly smaller MMILV $(P<0.05)$ and LV sizes $(P<0.01)$ than patients with the Pro/Pro genotype [Table 6].

Considering previous data that PPAR 2 affects gene expression in epithelial cells, vascular endothelium and macrophages, analysis of the state of blood vessels in different PPAR 2 genotypes was conducted [Table 6]. Analyzing the major vessels in patients with $\mathrm{EH}$ and concomitant DM2 showed that IMT in patients with the Pro12Ala/Ala12Ala genotype was significantly less $(P$ $<0.001)$ than in the Pro/Pro genotype. A significant difference $(P<0.05)$ was found in the PWV values of the CA depending on the PPAR 2 genotype. It was also established that in the main group of patients with the Pro/Pro genotype, the EDVD was significantly lower $(P<0.001)$ than in the Pro12Ala/Ala12Ala genotype. Established features of the differences of indicators in PPAR 2 genotypes confirm the association of PPAR 2 polymorphisms with the severity of endothelial dysfunction and vascular remodeling in patients with comorbidity of $\mathrm{EH}$ and DM2. 


\section{DISCUSSION}

Changes in echocardiographic parameters depending on genetic polymorphisms of the AGTR1 gene can be regarded as a result of varying activation of AT1 receptors, leading to differential expression and proliferation of cardiomyocytes and myocardium remodeling. ${ }^{[14,15]}$

The involvement of polymorphisms of the AGTR1 gene in the development and progression of atherosclerotic processes was demonstrated by significantly lower levels of anti-atherogenic high density lipoprotein cholesterol and significantly higher levels of glucose, $\mathrm{HbA} 1 \mathrm{c}$, insulin and HOMA-IR in patients with the $\mathrm{A} / \mathrm{C}$ $+C / C$ genotype compared to the $A / A$ genotype. More pronounced $\mathrm{IR}$ in the $A / C+C / C$ genotype can be explained by common mechanisms of hypertension and IR, including activation of the renin-angiotensinaldosterone system, which affects the sensitivity of tissues to insulin and compensatory hyperinsulinemia.

The differences in blood pressure with respect to PPAR 2 polymorphisms can be explained by the fact that the activity of PPAR 2 receptors also depends on the production of proinflammatory and hypertensive cytokines by adipose tissue, which leads to hypertension.

The influence of PPAR 2 polymorphisms in heart remodeling can be explained by the fact that PPAR 2 act as modulators of gene expression in many tissues, including smooth muscle, thus the alteration of their activity due to polymorphisms contributes to the development and progression of cardiovascular disease. ${ }^{[16-20]}$

More pronounced metabolic disturbances in the Pro/Pro genotype of PPAR 2 can be explained by the fact that $P P A R \gamma 2$ control adipogenesis (including the production of free fatty acids, elevated levels of which are the cause of IR), and activity of PPAR 2 affects production and circulation of lipoproteins and, as a consequence, the severity of atherosclerotic processes.

The modulating effect of polymorphisms of the genetic markers AGTR1 and PPAR 2 on the severity of cardiovascular remodeling in patients with comorbidity of EH and DM2 was, therefore, established.

In conclusion, polymorphisms of the genetic markers AGTR1 and PPAR 2 was associated with the development of comorbidity of $\mathrm{EH}$ and DM2. The $A / C$ and $C / C$ genotypes of the polymorphic marker A1166C of the AGTR1 gene were characterized by significantly higher blood pressure and more pronounced cardiovascular remodeling compared to the $A / A$ genotype. Patients with the Pro/Pro genotype of the Pro12Ala polymorphism of PPAR 2 had more severe hemodynamic and metabolic disorders.

\section{Authors' contributions}

A. Shalimova contributed solely to this paper.

\section{Financial support and sponsorship}

None.

\section{Conflicts of interest \\ There are no conflicts of interest.}

\section{Patient consent}

Each patient was informed the study and gave their consent.

\section{Ethics approval}

The study protocol was supported by the Ethics Committee of the Kharkiv National Medical University.

\section{REFERENCES}

1. Mettimano M, Romano-Spica V, Ianni A, Specchia M, Migneco A, Savi 1. AGT and AT1R gene polymorphism in hypertensive heart disease. In J Clin Pract 2002;56:574-7.

2. Martínez-Gómez LE, Cruz M, Martínez-Nava GA, Madrid-Marina V, Parra E, García-Mena J, Espinoza-Rojo M, Estrada-Velasco BI, Piza-Roma LF, Aquilera P, Burguete-García AI. A replication study of the IRS1, CAPN10, TCF7L2, and PPARG gene polymorphisms associated with type 2 diabetes in two different populations of Mexico. Ann Hum Genet 2011;75:612-20.

3. Czarnecka D, Kawecka-Jaszcz K, Stolarz K, Olszanecka A, Kieć-Wilk B, Dembińska-Kieć A. Genetic factors in hypertension. Angiotensinconverting enzyme polymorphism. Kardiol Pol 2004;61:1-10.

4. Wang X, Zhu H, Dong Y, Treiber FA, Snieder H. Effects of angiotensinogen and angiotensin II type I receptor genes on blood pressure and left ventricular mass trajectories in multiethnic youth. Twin Res Hum Genet 2006;9:393-402.

5. Mettimano M, Romano-Spica V, Ianni A, Specchia M, Migneco A, Savi L. AGT and AT1R gene polymorphism in hypertensive heart diseas. Int J Clin Pract 2002;56:574-7.

6. Berry C, Brosnan MJ, Fennell J, Hamilton CA, Dominiczak AF. Oxidative stress and vascular damage in hypertension. Curr Opin Nephrol Hypertens 2001;10:247-55.

7. de Denus S, Zakrzewski-Jakubiak M, Dubé MP, Bélanger F, Lepage S, Leblanc MH, Gossard D, Ducharme A, Racine N, Whittom L, Lavoie J, Touyz RM, Turgeon J, White M. Effects of AGTR1 A1166C gene polymorphism in patients with heart failure treated with candesartan. Ann Pharmacother 2008;42:925-32.

8. Grundy SM, Cleeman JI, Daniels SR, Donato KA, Eckel RH, Franklin BA, Gordon DJ, Krauss RM, Savage PJ, Smith SC Jr, Spertus JA, Costa F; America Heart Association; National Heart, Lung, and Blood Institue. Diagnosis and management of the metabolic syndrome: an American Heart Association/National Heart, Lung, and Blood Institue Scientific Statement. Circulation 2005;112:2735-52.

9. Colwell JA. Type 2 diabetes, Pre-diabetes, and the metabolic syndrome. JAMA 2011;306:215.

10. Saad MF, Rewers M, Selby J, Howard G, Jinagouda S, Fahmi S, 
Zaccaro D, Bergman RN, Savage PJ, Haffner SM. Insulin resistance and hypertension: the Insulin Resistance Atherosclerosis study. Hypertention 2004;43:1324-31.

11. Chen Z, Vigueira PA, Chambers KT, Hall AM, Mitra MS, Qi N, McDonald WG, Colca JR, Kletzien RF, Finck BN. Insulin resistance and metabolic derangements in obese mice are ameliorated by a novel peroxisome proliferator-activated receptor $\gamma$-sparing thiazolidinedione. J Biol Chem 2012;287:23537-48.

12. Yen CJ, Beamer BA, Negri C, Silver K, Brown KA, Yarnall DP, Burns DK, Roth J, Shuldiner AR. Molecular scanning of the human peroxisome proliferator activated receptor gamma (hPPAR gamma) gene in diabetic Caucasians: identification of a Pro12Ala PPAR gamma 2 missense mutation. Biochem Biophys Res Commun 1997;241:270-4.

13. Palatini P, Ceolotto G, Dorigatti F, Mos L, Santonastaso M, Bratti P, Papparella I, Pessina AC, Semplicini A. Angiotensin II type 1 receptor gene polymorphism predicts development of hypertension and metabolic syndrome. Am J Hypertens 2009;22:208-14.

14. Lehtonen J, Paukku K, Daviet L, Kontula K. Angiotensin II type 1 receptor 1166 polymorphism A to $\mathrm{C}$ increases mRNA stability and steady-state levels. Circulation 2006;114:190.
15. Jiang Z, Zhao W, Yu F, Xu G. Association of angiotension II type 1 receptor gene polymorphism with essentional hypertension. Chin Med $J$ (Engl) 2001;114:1249-51

16. Motavallian A, Andalib S, Vaseghi G, Mirmohammad-Sadeghi H, Amini M. Association between PRO12ALA polymorphism of the PPAR- $\gamma 2$ gene and type 2 diabetes mellitus in Iranian patients. Indian J Hum Genet 2013;19:239-44.

17. Ohshima K, Mogi M, Horiuchi M. Role of peroxisome proliferatoractivated receptor- $\gamma$ in vascular inflammation. Int $J$ Vasc Med 2012;2012:508416.

18. He W. PPAR 2 polymorphism and human health. PPAR Res 2009;2009:849538.

19. Zhang F, Lu Y, Zheng S. Peroxisome proliferator-activated receptor- $\gamma$ cross-regulation of signaling events implicated in liver fibrogenesis. Cell Signal 2012;24:596-605.

20. Gawrieh S, Marion MC, Komorowski R, Wallace J, Charlton M Kissebah A, Langefeld CD, Olivier M. Genetic variation in the peroxisome proliferator activated receptor-gamma gene is associated with histologically advanced NAFLD. Dig Dis Sci 2012;57:952-7. 